\title{
Fully coupled dual-porosity model for anisotropic formations
}

\author{
Ying Zhao ${ }^{\mathrm{a}, *}$, Mian Chen ${ }^{\mathrm{b}}$ \\ anstitute of Mechanics, Chinese Academy of Sciences, Beijing 100080, China \\ ${ }^{\mathrm{b}}$ School of Oil \& Gas Engineering, China University of Petroleum-Beijing, Beijing 102249, China
}

Accepted 2 March 2006

Available online 18 April 2006

\section{Introduction}

In recent decades, all petroleum reservoir problems involve two basic elements: fluid and rock. We are interested in two particular processes associated with them: fluid flow and geo-mechanics. Fluid flow is essential in a petroleum reservoir study. Geo-mechanics is believed to be important in several petroleum-engineering activities such as drilling, borehole stability, hydraulic fracturing and production-induced compaction and subsidence.

As we know, the production from naturally fractured reservoirs has a great potential worldwide, and many profitable reservoirs are naturally fractured. For naturally fractured reservoirs, economical petroleum production relies on the fracture permeability. The natural fractures basically are the product of the evolving rock stress state. Therefore, any disturbance of the stress field, such as due to fluid production/injection, can affect the existing fractures and the associated reservoir performance. A coupled fluidflow/ geo-mechanics model can provide a rational tool for a better understanding and management of naturally fractured reservoirs.

Naturally fractured reservoirs are often modeled by the dual-porosity type of concept developed by Barenblatt et al. [1] and Warren and Root [2]. Models incorporating both Biot's poroelastic theory [3] and Barrenblatt's dualporosity concept have been studied by several authors. The models they have established can be classified into two types, based on the approach taken.

One approach is based on the mixture theory, and was adopted by Wilson and Aifantis [4], Beskos and Aifantis [5], Bai et al. [6], and Berryman and Wang [7]. The resulting

\footnotetext{
${ }^{*}$ Corresponding author. Tel.: +86106263 4633; fax: + 861062561284 .

E-mail address: zhaoying@imech.ac.cn (Y. Zhao).
}

formulations have two related features: the first is that all the fluid-flow equations in a "mixture" have the same functional form as that of a single-porosity if the fluid exchange term is dropped; and the second is that phenomenological coefficients are proposed first, and their physical interpretations are deduced after the completion of the formulation. The first point implies that the stressdependent rock properties in one continuum are independent of the other mixing continua. This in turn may cause difficulty for the later physical interpretation (i.e., the second one) and even inconsistency with the geo-mechanical equations adopted.

The other approach follows the route of conventional fluid-flow modeling. Coupling of geo-mechanics is identified through stress-dependent rock properties and during the development process. Interpretation of the stressdependent properties is therefore critical to achieving a proper coupling. This approach was adopted by Duguid and Lee [8], Valliappan and Khalili-Naghadeh [9], KhaliliNaghadeh and Valliappan [10], Chen et al. [11], and Li et al. [12].

In the model of Duguid and Lee [8], an incompressible solid was assumed. Also, no explicit rock compressibilities (solid, pore, and bulk) appear in their fluid-flow equations. Explicit rock compressibilities were considered by Valliappan and Khalili-Naghadeh [9] (also [10]). But except for the case of an incompressible solid, their two fluid pressure equations do not collapse to the corresponding singleporosity equation when the two fluid pressures reach equilibrium (i.e., $p_{1}=p_{2}$ ). So it suggests that incompressible solid phase had been implicitly adopted in their general derivations which, however, contradicts their intention and the presented equations.

The disadvantages identified in the above models are resolved in the model of $\mathrm{Li}$ et al. [12]. Specifically, (i) internal model consistency is maintained, and 
(ii) continuity between the single-porosity and dualporosity systems can be established smoothly.

The above models are all based on the assumption of isotropy, whereas most rocks are characterized by anisotropy of various degrees. Therefore, research on the behavior of anisotropic dual-porosity media is of relevance to oil and gas production. This paper is focused on the development of the governing equations for a fully coupled dual-porosity model for anisotropic rock formations.

\section{Geo-mechanical model}

The three basic principles of poroelastic theory are: stress equilibrium, strain-displacement, and strain-stresspressure relations. Mathematically, these are the staticequilibrium equation:

$\frac{\partial \sigma_{i j}}{\partial x_{j}}=0$

the strain-displacement relation:

$\varepsilon_{i j}=\frac{1}{2}\left(\frac{\partial u_{i}}{\partial x_{j}}+\frac{\partial u_{j}}{\partial x_{i}}\right)$

and the effective stress principle and the stress-strain constitutive relation for anisotropic double porous media (strain-stress-pressure relations):

$\sigma_{i j}^{\mathrm{e}}=\sigma_{i j}-\alpha_{i j}^{1} p_{1}-\alpha_{i j}^{2} p_{2}=C_{i j k l} \varepsilon_{k l}$.

In Eqs. (1)-(3), $\sigma_{i j}$ and $\varepsilon_{k l}$ are the components of total stress tensor and bulk strain tensor, respectively. We should note that the sign convention here follows that in which compressive stresses are positive. Additionally, $u_{i}$ is the solid displacement vector, $C_{i j k l}$ denotes the elastic moduli tensor, $\sigma_{i j}^{\mathrm{e}}$ denotes the effective stress tensor, $p_{n}$ is the $n$th fluid pressure, $\alpha_{i j}^{n}$ is the $n$th Biot coefficient tensor (where the subscript $n=1,2$ indicates the matrix-block and fractures, respectively). For anisotropic double porous media, $\alpha_{i j}^{n}$ has the following form [13]:

$\alpha_{i j}^{1}=\delta_{i j}-C_{i j k l} M_{k l m n}^{*}$,

$\alpha_{i j}^{2}=C_{i j k l} M_{k l m n}^{*}-C_{i j k l} M_{k l m n}^{s}$,

where $\delta_{i j}$ is Kronecker's delta $\left(\delta_{i j}=1\right.$ for $i=j, \delta_{i j}=0$ for $i \neq j), M_{k l m n}$ is the elastic compliance tensor, the superscript * stands for a porous media of without fractures, and the subscripts $s$ represents solid. Body forces and inertial effects are neglected in Eq. (1). Small strains are implied in Eq. (2). Substituting Eq. (3) into the equilibrium Eq. (1) gives

$\frac{1}{2} C_{i j k l}\left(\frac{\partial^{2} u_{k}}{\partial x_{j} \partial x_{l}}+\frac{\partial^{2} u_{l}}{\partial x_{j} \partial x_{k}}\right)=-\alpha_{i j}^{1} \frac{\partial p_{1}}{\partial x_{j}}-\alpha_{i j}^{2} \frac{\partial p_{2}}{\partial x_{j}}$.

Eq. (5) gives the geo-mechanical model for anisotropic double porous media. Three simplifications of the geomechanical model are discussed next.

\subsection{Transverse isotropy}

The geo-mechanical model Eq. (5) becomes more tractable in the case of transverse isotropy. This is an important type of anisotropy in geophysical applications, since material properties are frequently isotropic in the bedding plane but differ in the direction normal to this plane. The anisotropy may be either structural (anisotropic pore geometry), intrinsic (anisotropic solid material), or both.

The tensor of elastic moduli for a transversely isotropic material has the form [14]:

$$
\begin{aligned}
C_{i j k l}= & \mu\left(\delta_{i k} \delta_{j l}+\delta_{i l} \delta_{j k}\right)+\lambda \delta_{i j} \delta_{k l}+\alpha\left(\delta_{i k} h_{j} h_{l}+\delta_{j l} h_{i} h_{k}\right. \\
& \left.+\delta_{i l} h_{j} h_{k}+\delta_{j k} h_{i} h_{l}\right)+\beta\left(\delta_{i j} h_{k} h_{l}+\delta_{k l} h_{i} h_{j}\right)+\gamma h_{i} h_{j} h_{k} h_{l},
\end{aligned}
$$

where $\alpha, \beta, \gamma, \lambda$, and $\mu$ are constants, and $h_{i}$ is the directional cosine of symmetry axis. For isotropic materials, $\alpha, \beta$ and $\gamma$ vanish; $\lambda$ and $\mu$ are the Lamé constants. Introducing Eq. (6) into Eq. (5) give

$$
\begin{aligned}
& \mu \frac{\partial^{2} u_{i}}{\partial x_{j} \partial x_{j}}+\alpha h_{i} h_{k} \frac{\partial^{2} u_{k}}{\partial x_{j} \partial x_{j}}+(\mu+\lambda) \frac{\partial^{2} u_{j}}{\partial x_{j} x_{i}} \\
& +(\alpha+\beta) h_{i} h_{j} \frac{\partial^{2} u_{k}}{\partial x_{j} \partial x_{k}}+\alpha h_{j} h_{k} \frac{\partial^{2} u_{i}}{\partial x_{j} \partial x_{k}}+(\alpha+\beta) h_{j} h_{k} \frac{\partial^{2} u_{k}}{\partial x_{j} \partial x_{i}} \\
& +\gamma h_{i} h_{j} h_{k} h_{l} \frac{\partial^{2} u_{k}}{\partial x_{j} \partial x_{l}}=-\alpha_{i j}^{1} \frac{\partial p_{1}}{\partial x_{j}}-\alpha_{i j}^{2} \frac{\partial p_{2}}{\partial x_{j}}
\end{aligned}
$$

where the Biot coefficient tensors for a transversely isotropic material have the forms [13]

$$
\begin{aligned}
\alpha_{i j}^{1}= & \delta_{i j}\left(1-A A_{1}^{*}-C B_{1}^{*}\right)-\left(B A_{1}^{*}+D B_{1}^{*}\right) h_{i} h_{j}, \\
\alpha_{i j}^{2}= & \delta_{i j}\left(A A_{1}^{*}+C B_{1}^{*}-A A_{1}^{\mathrm{s}}-C B_{1}^{\mathrm{s}}\right) \\
& +\left(B A_{1}^{*}+D B_{1}^{*}-B A_{1}^{\mathrm{s}}-D B_{1}^{\mathrm{s}}\right) h_{i} h_{j}
\end{aligned}
$$

and

$$
\begin{aligned}
A & =2 \mu+3 \lambda+\beta, \quad B=4 \alpha+3 \beta+\gamma, \\
C & =\lambda+\beta, \quad D=2 \mu+4 \alpha+\beta+\gamma, \\
A_{1}^{\mathrm{s}} & =2 \mu_{1}^{s}+3 \lambda_{1}^{\mathrm{s}}+\beta_{1}^{\mathrm{s}}, \quad B_{1}^{\mathrm{s}}=4 \alpha_{1}^{\mathrm{s}}+3 \beta_{1}^{\mathrm{s}}+\gamma_{1}^{\mathrm{s}}, \\
A_{1}^{*} & =2 \mu_{1}^{*}+3 \lambda_{1}^{*}+\beta_{1}^{*}, \quad B_{1}^{*}=4 \alpha_{1}^{*}+3 \beta_{1}^{*}+\gamma_{1}^{*} .
\end{aligned}
$$

The compliance tensor has the similar format as the above elastic modulus tensor, with compliance coefficients $\mu_{1}, \lambda_{1}, \alpha_{1}, \beta_{1}$ and $\gamma_{1}$, which can be expressed in terms of the moduli $\mu, \lambda, \alpha, \beta$ and $\gamma$.

\subsection{Structural anisotropy}

The geo-mechanical model described by Eq. (7) is simplified considerably when the anisotropy is structural rather than intrinsic, i.e., in the case of an isotropic solid material with an anisotropic pore structure. If the anisotropy is purely structural, so that the solid material is isotropic, then the Biot coefficient tensors Eq. (8) 
reduce to [13]

$\alpha_{i j}^{1}=\delta_{i j}\left(1-A A_{1}^{*}-C B_{1}^{*}\right)-\left(B A_{1}^{*}+D B_{1}^{*}\right) h_{i} h_{j}$,

$\alpha_{i j}^{2}=\delta_{i j}\left(A A_{1}^{*}+C B_{1}^{*}-\frac{A c_{\mathrm{s}}}{3}\right)+\left(B A_{1}^{*}+D B_{1}^{*}-\frac{B c_{\mathrm{s}}}{3}\right) h_{i} h_{j}$,

where $c_{\mathrm{s}}$ represents the unjacketed bulk compressibility of the fractured rock.

\subsection{Isotropy}

If the pore geometry is also isotropic, then Eq. (7) and (9) reduce to, respectively:

$\mu \frac{\partial^{2} u_{i}}{\partial x_{j} \partial x_{j}}+(\mu+\lambda) \frac{\partial e}{\partial x_{i}}=-\alpha_{i j}^{1} \frac{\partial p_{1}}{\partial x_{j}}-\alpha_{i j}^{2} \frac{\partial p_{2}}{\partial x_{j}}$,

$\alpha_{i j}^{1}=\delta_{i j}\left(1-\frac{c^{*}}{c_{\mathrm{b}}}\right), \alpha_{i j}^{2}=\delta_{i j}\left(\frac{c^{*}-c_{\mathrm{s}}}{c_{\mathrm{b}}}\right)$

in which $c_{\mathrm{b}}$ is the drained jacketed bulk compressibility of the fractured rock, $c^{*}$ is the compressibility coefficient for porous media without fractures, $e$ is the dilatation or volume strain of the solid skeleton which is related to solid displacement vector $\mathbf{u}$ by $e=\operatorname{div} \mathbf{u}$. Introducing Eq. (11) into Eq. (10) gives

$\mu \frac{\partial^{2} u_{i}}{\partial x_{j} \partial x_{j}}+(\mu+\lambda) \frac{\partial e}{\partial x_{i}}=-\alpha_{1} \frac{\partial p_{1}}{\partial x_{i}}-\alpha_{2} \frac{\partial p_{2}}{\partial x_{i}}$,

where $\alpha_{1}=1-\left(c^{*} / c_{\mathrm{b}}\right), \alpha_{2}=\left(c^{*}-c_{\mathrm{s}}\right) / c_{\mathrm{b}}$. Eq. (12) is the geo-mechanical model for isotropic double porous media with linear elastic deformation, the same as that presented in [12].

\section{Fluid flow model}

The three basic principles of fluid flow in porous media are mass conservation, Darcy's law, and the equation of state. The mass conservation equations take the form

Fluid status : $\left(\rho \phi_{n} v_{n i f}\right)_{, i}+\frac{\partial\left(\phi_{n} \rho\right)}{\partial t}+(-1)^{n} \rho \Gamma=0$.

Solid status : $\left[\rho_{\mathrm{s}}\left(1-\phi_{\mathrm{t}}\right) v_{i \mathrm{~s}}\right]_{, i}+\frac{\partial\left[\left(1-\phi_{\mathrm{t}}\right) \rho_{\mathrm{s}}\right]}{\partial t}=0$.

Darcy's law for fluid flow can be written as

$v_{n i}=\phi_{n}\left(v_{n i \mathrm{f}}-v_{i \mathrm{~s}}\right)=-\frac{k_{i j}^{n}}{\mu} p_{n, j}$

The equation of state (isothermal fluid compressibility) is

$c_{n}=\frac{1}{\rho} \frac{\partial \rho}{\partial p_{n}}$.

In Eqs. (13)-(16), $\rho$ is the fluid density, which is assumed to be a function of fluid pressure only, i.e., $\rho \equiv \rho(p), v_{n i f}$ is the $n$th fluid velocity vector, $\phi_{n}$ is the $n$th effective porosity, $\phi_{\mathrm{t}}$ is the total effective porosity (i.e., $\phi_{\mathrm{t}}=\phi_{1}+\phi_{2}$, $\left.1=\phi_{1}+\phi_{2}+\left(V_{\mathrm{s}} / V_{\mathrm{b}}\right)\right)$. The solid counterparts for density and velocity are $\rho_{\mathrm{s}}$ and $v_{i s}, v_{n i}$ is the seepage velocity, $\mu$ is the fluid viscosity, which is also assumed to be a function of fluid pressure only, i.e., $\mu \equiv \mu(p) . p_{n, j}$ is the $n$th fluid pressure gradient, $c_{n}$ is the $n$th fluid compressibility, and $t$ is the time. $k_{i j}^{n}$ is the $n$th permeability tensor, and anisotropy of the permeability is considered. $\Gamma$ is the interporosity-flow term which represents the volumetric exchange rate between fractures and matrix blocks per unit bulk volume $\left(V_{\mathrm{b}}=V_{p 1}+V_{p 2}+V_{\mathrm{s}}\right) . \Gamma$ is a unique aspect of the dual-porosity concept. However, $\Gamma$ is also one of the major uncertainties in any dual-porosity-based (also multiporosity-based) model. The uncertainty is primarily due to the difficulty in describing the configuration, distribution, and connectivity of the fracture. In the most general form, $\Gamma$ is a function of the two fluid-pressure fields (matrixblocks and fractures) and the reservoir stress field. Descriptions of $\Gamma$ may range from a simple pressuredifference-controlled mechanism, i.e., $\Gamma \equiv \Gamma\left(p_{1}-p_{2}\right)[1,2]$, to a more complicated history-dependent mechanism [15]. Because of the inherent uncertainty of $\Gamma$ and the emphasis of this study to incorporate the geo-mechanics into a dualporosity concept, we will not specify the exact functional form of $\Gamma$ in this paper other than making the above general comments.

Introducing Darcy's law Eq. (15) into Eq. (13) gives

$\left(\rho \frac{k_{i j}^{n}}{\mu} p_{n, j}\right)_{, i}=\frac{\mathrm{d}\left(\phi_{n} \rho\right)}{\mathrm{d} t}+\phi_{n} \rho\left(v_{i s}\right)_{, i}+(-1)^{n} \rho \Gamma$,

where $\mathrm{d}(\cdot) / \mathrm{d} t$ is the material derivative with respect to a moving solid, defined as

$\frac{\mathrm{d}(\cdot)}{\mathrm{d} t}=\frac{\partial(\cdot)}{\partial t}+\mathbf{v}_{\mathrm{s}} \cdot \nabla(\cdot)$.

Note that for nondeformable media the material derivative is equivalent to a partial derivative, since $\mathbf{v}_{\mathrm{s}}=0$.

Expanding the right-hand side of Eq. (17) results in

$\left(\rho \frac{k_{i j}^{n}}{\mu} p_{n, j}\right)_{, i}=\phi_{n} \rho\left[\frac{1}{\rho} \frac{\mathrm{d} \rho}{\mathrm{d} t}+\frac{1}{\phi_{n}} \frac{\mathrm{d} \phi_{n}}{\mathrm{~d} t}+\left(v_{i s}\right)_{, i}\right]+(-1)^{n} \rho \Gamma$.

Expanding the first term of the solid mass balance equation, Eq. (14), gives

$\left(v_{i \mathrm{~s}}\right)_{, i}=-\frac{1}{\rho_{\mathrm{s}}\left(1-\phi_{\mathrm{t}}\right)} \frac{\mathrm{d}\left[\left(1-\phi_{\mathrm{t}}\right) \rho_{\mathrm{s}}\right]}{\mathrm{d} t}=\frac{1}{V_{\mathrm{b}}} \frac{\mathrm{d} V_{\mathrm{b}}}{\mathrm{d} t}$.

Thus, the divergence of solid velocity simply reflects the rate of change of bulk volume. According to Eq. (20), $\mathbf{v}_{\mathrm{s}}=$ 0 (non-deformable solid) implies $\mathrm{d} V_{\mathrm{b}}=0$.

According to the relation $\phi_{n}=V_{p n} / V_{\mathrm{b}}$, we have

$\frac{\mathrm{d} \phi_{n}}{\phi_{n}}=\frac{\mathrm{d} V_{p n}}{V_{p n}}-\frac{\mathrm{d} V_{\mathrm{b}}}{V_{\mathrm{b}}}$.

Introducing Eqs. (20), (21) into (19) results in

$\left(\rho \frac{k_{i j}^{n}}{\mu} p_{n, j}\right)_{, i}=\phi_{n} \rho\left[\frac{1}{\rho} \frac{\mathrm{d} \rho}{\mathrm{d} t}+\frac{1}{V_{p n}} \frac{\mathrm{d} V_{p n}}{\mathrm{~d} t}\right]+(-1)^{n} \rho \Gamma$. 
Note that the right-hand-side of Eq. (22) basically represents the rate of change of fluid density and pore volume. The change of fluid density is related to the fluid compressibility $c_{n}$ by the following relations (Eq. (16)):

$c_{n} \frac{\partial p_{n}}{\partial t}=\frac{1}{\rho} \frac{\partial \rho}{\partial t} ; c_{n} \nabla p_{n}=\frac{1}{\rho} \nabla \rho ; c_{n} \frac{\mathrm{d} p_{n}}{\mathrm{~d} t}=\frac{1}{\rho} \frac{\mathrm{d} \rho}{\mathrm{d} t}$

Since the fluid density is a function of fluid pressure only, interpretation of the volumetric change of pore volume thus is crucial in coupling fluid-flow and geo-mechanics.

Introducing above equation into Eq. (22) gives

$$
\left(\rho \frac{k_{i j}^{n}}{\mu} p_{n, j}\right)_{, i}=\phi_{n} \rho\left[c_{n} \frac{\mathrm{d} p_{n}}{\mathrm{~d} t}+\frac{1}{V_{p n}} \frac{\mathrm{d} V_{p n}}{\mathrm{~d} t}\right]+(-1)^{n} \rho \Gamma
$$

Consider now the change of pore volume term $\mathrm{d} V_{p n} / V_{p n}$ in Eq. (24). Obviously, as a result of the increasing of the fluid pressure $p_{n}$, the pore volume $V_{p n}$ will increase. And the increasing of the applied stress can decrease both the skeleton volume and the pore volume. Now one representative element of dual-porosity media is considered, which must include a sufficient number of solid grains, matrix pores, and fractures. Through the Betti's reciprocal theorem of elasticity, three relations are concluded:

$$
\begin{aligned}
& \mathrm{d} \sigma_{i j} M_{i j k k}^{*} \mathrm{~d} p_{1}+\mathrm{d} p_{1} \phi_{1} M_{i i k k}^{*} \mathrm{~d} p_{1}+\mathrm{d} p_{2} \phi_{2} \varepsilon_{i i}^{*} \\
& \quad=\mathrm{d} p_{1} M_{i i k l} \mathrm{~d} \sigma_{k l}-\mathrm{d} p_{1}\left(M_{i i k k}-M_{i i k k}^{*}\right) \mathrm{d} p_{1} \\
& \quad+\mathrm{d} p_{2}\left(M_{i i k k}^{\mathrm{s}}-M_{i i k k}^{*}\right) \mathrm{d} p_{1}+\mathrm{d} p_{1} \phi_{1} \varepsilon_{i i}^{1}, \\
& M_{i i k l} \mathrm{~d} \sigma_{k l}-\mathrm{d} p_{1}\left(M_{i i k k}-M_{i i k k}^{*}\right)+\left(M_{i i k k}^{\mathrm{s}}-M_{i i k k}^{*}\right) \mathrm{d} p_{2} \\
& \quad+\varepsilon_{i i}^{1} \phi_{1}+\varepsilon_{i i}^{2} \phi_{2}=\mathrm{d} \sigma_{i j} M_{i j k k}^{\mathrm{s}}+\mathrm{d} p_{1} \phi_{1} M_{i i k k}^{\mathrm{s}}+\mathrm{d} p_{2} \phi_{2} M_{i i k k,}^{\mathrm{s}},
\end{aligned}
$$

$\mathrm{d} p_{1} M_{i i k k}^{\mathrm{s}}+\mathrm{d} p_{1} \phi_{1} M_{i i k k}^{\mathrm{s}}=M_{i i k k}^{*} \mathrm{~d} p_{1}+\phi_{1} M_{i i k k}^{*} \mathrm{~d} p_{1}+\phi_{2} \varepsilon_{i i}^{*}$,

where $\varepsilon_{i i}$ is the volumetric strain, the meaning of superscripts 1,2 and $*$ are the same as mentioned above, and the repeated indices imply summation. From the above Eq. (27), we find

$\phi_{2} \varepsilon_{i i}^{*}=\mathrm{d} p_{1}\left(1+\phi_{1}\right)\left(M_{i i k k}^{\mathrm{s}}-M_{i i k k}^{*}\right)$.

Eliminating $\phi_{2} \varepsilon_{i i}^{*}$ between Eqs. (28) and (25), and applying the definition of volumetric strain, results in

$$
\begin{aligned}
& \frac{\mathrm{d} V_{p_{1}}}{V_{p_{1}}} \phi_{1}=\varepsilon_{i i}^{1} \phi_{1}=\mathrm{d} \sigma_{i j}\left(M_{i j k k}^{*}-M_{k k i j}\right) \\
& \quad+\mathrm{d} p_{1}\left[\phi_{1} M_{i i k k}^{*}+\left(M_{i i k k}-M_{i i k k}^{*}\right)\right]+\mathrm{d} p_{2} \phi_{1}\left(M_{i k k}^{\mathrm{s}}-M_{i i k k}^{*}\right),
\end{aligned}
$$

$$
\begin{aligned}
& \frac{\mathrm{d} V_{p_{2}}}{V_{p_{2}}} \phi_{2}=\varepsilon_{i i}^{2} \phi_{2}=\mathrm{d} \sigma_{i j}\left(M_{i j k k}^{\mathrm{s}}-M_{i j k k}^{*}\right) \\
& \quad+\mathrm{d} p_{1} \phi_{1}\left(M_{i i k k}^{\mathrm{s}}-M_{i i k k}^{*}\right)+\mathrm{d} p_{2}\left[\phi_{2} M_{i i k k}^{\mathrm{s}}-\left(1+\phi_{1}\right)\left(M_{i i k k}^{\mathrm{s}}-M_{i i k k}^{*}\right)\right],
\end{aligned}
$$

where $V_{p_{n}}$ is the $n$th pore volume. Introducing Eqs. (29) and (30) into the Eq. (24) results in

$$
\begin{gathered}
\left(\frac{k_{i j}^{1}}{\mu} p_{1, j}\right)_{, i}=, a_{11} \frac{\partial p_{1}}{\partial t}+a_{12} \frac{\partial p_{2}}{\partial t}+a_{13 i j} \frac{\partial \sigma_{i j}}{\partial t}-\Gamma, \\
\left(\frac{k_{i j}^{2}}{\mu} p_{2, j}\right)_{, i}=a_{21} \frac{\partial p_{1}}{\partial t}+a_{22} \frac{\partial p_{2}}{\partial t}+a_{23 i j} \frac{\partial \sigma_{i j}}{\partial t}+\Gamma
\end{gathered}
$$

in which

$$
\begin{aligned}
a_{11} & =\phi_{1}\left(M_{i i k k}^{*}+c_{1}\right)+\left(M_{i i k k}-M_{i i k k}^{*}\right), \\
a_{12} & =a_{21}=\phi_{1}\left(M_{i i k k}^{\mathrm{s}}-M_{i i k k}^{*}\right), \\
a_{13 i j} & =M_{i j k k}^{*}-M_{i j k k}, \\
a_{22} & =\phi_{2}\left(M_{i i k k}^{\mathrm{s}}+c_{2}\right)-\left(1+\phi_{1}\right)\left(M_{i i k k}^{\mathrm{s}}-M_{i i k k}^{*}\right), \\
a_{23 i j} & =M_{i j k k}^{\mathrm{s}}-M_{i j k k}^{*} .
\end{aligned}
$$

Note that $a_{13 i j}$ and $a_{23 i j}$ are not scalars, but are tensors.

Two assumptions have been imposed in deriving Eq. (31). First, the material derivatives can be approximated by the partial derivative, i.e., $\mathrm{d}(\cdot) / \mathrm{d} t \cong \partial(\cdot) / \partial t$. This is equivalent to neglecting the dot product term in Eq. (18) by assuming $\mathbf{v}_{\mathrm{s}} \cdot \nabla(\cdot) \ll \partial(\cdot) / \partial t$. Second, the quadratic term $c_{n}(\nabla p)^{2}$ is negligible compared to the other terms.

Introducing the Eqs. (2) and (3) into Eq. (31) results in:

$$
\begin{aligned}
& \left(\frac{k_{i j}^{1}}{\mu} p_{1, j}\right)_{, i}=b_{11} \frac{\partial p_{1}}{\partial t}+b_{12} \frac{\partial p_{2}}{\partial t}-\frac{1}{2} \alpha_{i j}^{1} \frac{\partial}{\partial t}\left(\frac{\partial u_{i}}{\partial x_{j}}+\frac{\partial u_{j}}{\partial x_{i}}\right)-\Gamma, \\
& \left(\frac{k_{i j}^{2}}{\mu} p_{2, j}\right)_{, i}=b_{21} \frac{\partial p_{1}}{\partial t}+b_{22} \frac{\partial p_{2}}{\partial t}-\frac{1}{2} \alpha_{i j}^{2} \frac{\partial}{\partial t}\left(\frac{\partial u_{i}}{\partial x_{j}}+\frac{\partial u_{j}}{\partial x_{i}}\right)+\Gamma
\end{aligned}
$$

in which

$$
\begin{aligned}
& b_{11}=\phi_{1}\left(M_{i i k k}^{*}+c_{1}\right)+\left(M_{i i k k}-M_{i i k k}^{*}\right)+a_{13 i j} \alpha_{i j}^{1}, \\
& b_{12}=\phi_{1}\left(M_{i i k k}^{\mathrm{s}}-M_{i i k k}^{*}\right)+a_{13 i j} a_{i j}^{2}, \\
& b_{21}=\phi_{1}\left(M_{i i k k}^{\mathrm{s}}-M_{i i k k}^{*}\right)+a_{23 i j} a_{i j}^{1}, \\
& b_{22}=\phi_{2}\left(M_{i i k k}^{\mathrm{s}}+c_{2}\right)-\left(1+\phi_{1}\right)\left(M_{i i k k}^{\mathrm{s}}-M_{i i k k}^{*}\right)+a_{23 i j} \alpha_{i j}^{2},
\end{aligned}
$$

where $\alpha_{i j}^{1}$ and $\alpha_{i j}^{2}$ are defined in Eq. (4), and $\alpha_{13 i j}$ and $\alpha_{23 i j}$ are defined in Eq. (32).

Three simplifications of the fluid-flow model for anisotropic double porous media are discussed next.

\subsection{Transverse isotropy}

The tensor of elastic moduli for a transversely isotropic material has the form [14]

$$
\begin{aligned}
M_{i j k l}=\mu_{1} & \left(\delta_{i k} \delta_{j l}+\delta_{i l} \delta_{j k}\right)+\lambda_{1} \delta_{i j} \delta_{k l} \\
& +\alpha_{1}\left(\delta_{i k} h_{j} h_{l}+\delta_{j l} h_{i} h_{k}+\delta_{i l} h_{j} h_{k}+\delta_{j k} h_{i} h_{l}\right) \\
& +\beta_{1}\left(\delta_{i j} h_{k} h_{l}+\delta_{k l} h_{i} h_{j}\right)+\gamma_{1} h_{i} h_{j} h_{k} h_{l} .
\end{aligned}
$$

Then:

$$
M_{i j k k}=M_{k k i j}=A_{1} \delta_{i j}+B_{1} h_{i} h_{j}, \quad M_{i i k k}=3 A_{1}+B_{1},
$$


where $A_{1}=2 \mu_{1}+3 \lambda_{1}+\beta_{1}, \quad B_{1}=4 \alpha_{1}+3 \beta_{1}+\gamma_{1}$. The equations analogous to Eq. (36) now give

$$
\begin{aligned}
& M_{i j k k}^{\mathrm{s}}=A_{1}^{\mathrm{s}} \delta_{i j}+B_{1}^{\mathrm{s}} h_{i} h_{j}, \quad M_{i i k k}^{\mathrm{s}}=3 A_{1}^{\mathrm{s}}+B_{1}^{\mathrm{s}}, \\
& M_{i j k k}^{*}=A_{1}^{*} \delta_{i j}+B_{1}^{*} h_{i} h_{j}, \quad M_{i i k k}^{*}=3 A_{1}^{*}+B_{1}^{*} .
\end{aligned}
$$

Introducing Eqs. (36) and (37) into Eq. (34) results in:

$$
\begin{aligned}
b_{11}= & 3 \phi_{1} A_{1}^{*}+\phi_{1} B_{1}^{*}+\phi_{1} c_{1}+\left(A A_{1}^{*}+C B_{1}^{*}\right) \\
& \left(3 A_{1}+B_{1}-3 A_{1}^{*}-B_{1}^{*}\right) \\
& +\left(A_{1}+B_{1}-A_{1}^{*}-B_{1}^{*}\right)\left(B A_{1}^{*}+D B_{1}^{*}\right), \\
b_{12}= & 3 \phi_{1} A_{1}^{\mathrm{s}}+\phi_{1} B_{1}^{\mathrm{s}}-3 \phi_{1} A_{1}^{*}-\phi_{1} B_{1}^{*} \\
& -\left(3 A_{1}+B_{1}-3 A_{1}^{*}-B_{1}^{*}\right)\left(A A_{1}^{*}+C B_{1}^{*}-A A_{1}^{\mathrm{s}}-C B_{1}^{\mathrm{s}}\right) \\
& -\left(A_{1}+B_{1}-A_{1}^{*}-B_{1}^{*}\right)\left(B A_{1}^{*}+D B_{1}^{*}-B A_{1}^{\mathrm{s}}-D B_{1}^{\mathrm{s}}\right), \\
b_{21}= & 3 \phi_{1} A_{1}^{s}+\phi_{1} B_{1}^{\mathrm{s}}-3 \phi_{1} A_{1}^{*}-\phi_{1} B_{1}^{*} \\
& +\left(3 A_{1}^{s}+B_{1}^{s}-3 A_{1}^{*}-B_{1}^{*}\right)\left(1-A A_{1}^{*}-C B_{1}^{*}\right) \\
& -\left(A_{1}^{\mathrm{s}}+B_{1}^{\mathrm{s}}-A_{1}^{*}-B_{1}^{*}\right)\left(B A_{1}^{*}+D B_{1}^{*}\right), \\
b_{22}= & 3\left(\phi_{2}-\phi_{1}-1\right) A_{1}^{\mathrm{s}}+\left(\phi_{2}-\phi_{1}-1\right) B_{1}^{\mathrm{s}}+3\left(1+\phi_{1}\right) A_{1}^{*} \\
& +\left(1+\phi_{1}\right) B_{1}^{*}+\phi_{2} c_{2}+\left(3 A_{1}^{\mathrm{s}}+B_{1}^{\mathrm{s}}-3 A_{1}^{*}-B_{1}^{*}\right) \\
& \left(A A_{1}^{*}+C B_{1}^{*}-A A_{1}^{\mathrm{s}}-C B_{1}^{\mathrm{s}}\right) \\
& +\left(A_{1}^{\mathrm{s}}+B_{1}^{\mathrm{s}}-A_{1}^{*}-B_{1}^{*}\right)\left(B A_{1}^{*}+D B_{1}^{*}-B A_{1}^{\mathrm{s}}-D B_{1}^{\mathrm{s}}\right)
\end{aligned}
$$

and $\alpha_{i j}^{1}$ and $\alpha_{i j}^{2}$ in Eq. (33) are reduced to Eq. (8).

\subsection{Structural anisotropy}

When the anisotropy is structural rather than intrinsic, i.e., in the case of an isotropic solid material with an anisotropic pore structure, we have

$A_{1}^{\mathrm{s}}=\frac{1}{A^{\mathrm{s}}}=\frac{1}{2 \mu+3 \lambda}=\frac{c_{\mathrm{s}}}{3}, \quad B_{1}^{\mathrm{s}}=B^{\mathrm{s}}=0$.

Introducing Eq. (39) into Eq. (38) gives

$$
\begin{aligned}
b_{11}= & 3 \phi_{1} A_{1}^{*}+\phi_{1} B_{1}^{*}+\phi_{1} c_{1}+\left(A A_{1}^{*}+C B_{1}^{*}\right) \\
& \times\left(3 A_{1}+B_{1}-3 A_{1}^{*}-B_{1}^{*}\right)+\left(A_{1}+B_{1}-A_{1}^{*}-B_{1}^{*}\right) \\
& \times\left(B A_{1}^{*}+D B_{1}^{*}\right), \\
b_{12}= & \phi_{1} c_{\mathrm{s}}-3 \phi_{1} A_{1}^{*}-\phi_{1} B_{1}^{*} \\
& -\left(3 A_{1}+B_{1}-3 A_{1}^{*}-B_{1}^{*}\right)\left(A A_{1}^{*}+C B_{1}^{*}-A c_{\mathrm{s}} / 3\right) \\
& -\left(A_{1}+B_{1}-A_{1}^{*}-B_{1}^{*}\right)\left(B A_{1}^{*}+D B_{1}^{*}-B c_{\mathrm{s}} / 3\right), \\
b_{21}= & \phi_{1} c_{\mathrm{s}}-3 \phi_{1} A_{1}^{*}-\phi_{1} B_{1}^{*}+\left(c_{\mathrm{s}}-3 A_{1}^{*}-B_{1}^{*}\right) \\
& \left(1-A A_{1}^{*}-C B_{1}^{*}\right)-\left(c_{\mathrm{s}}-A_{1}^{*}-B_{1}^{*}\right)\left(B A_{1}^{*}+D B_{1}^{*}\right), \\
b_{22}= & \left(\phi_{2}-\phi_{1}-1\right) c_{\mathrm{s}}+3\left(1+\phi_{1}\right) A_{1}^{*}+\left(1+\phi_{1}\right) B_{1}^{*}+\phi_{2} c_{2} \\
& +\left(c_{\mathrm{s}}-3 A_{1}^{*}-B_{1}^{*}\right)\left(A A_{1}^{*}+C B_{1}^{*}-A c_{\mathrm{s}} / 3\right) \\
& +\left(c_{\mathrm{s}} / 3-A_{1}^{*}-B_{1}^{*}\right)\left(B A_{1}^{*}+D B_{1}^{*}-B c_{\mathrm{s}} / 3\right)
\end{aligned}
$$

and $\alpha_{i j}^{1}$ and $\alpha_{i j}^{2}$ in Eq. (33) are reduced to Eq. (9).

\subsection{Isotropy}

If the pore geometry is also isotropic, then equations analogous to Eq. (39) hold for the porous material also:

$A_{1}^{*}=\frac{c^{*}}{3}, \quad A_{1}=\frac{1}{A}=\frac{c_{\mathrm{b}}}{3}, \quad B_{1}^{*}=B=B_{1}=0$

and Eq. (40) reduces to

$b_{11}=-\alpha_{1} \alpha_{1} c_{\mathrm{b}}+\alpha_{1} c_{\mathrm{b}}\left(1-\phi_{1}\right)+\left(c_{\mathrm{b}}+c_{1}\right) \phi_{1}$,

$b_{12}=b_{21}=-\alpha_{1} \alpha_{2} c_{\mathrm{b}}-\alpha_{2} c_{\mathrm{b}} \phi_{1}$,

$b_{22}=-\alpha_{2} \alpha_{2} c_{\mathrm{b}}+\alpha_{2} c_{\mathrm{b}}\left(1+\phi_{1}\right)+\phi_{2}\left(c_{2}+c_{\mathrm{s}}\right)$,

where $\alpha_{1}=1-\left(c^{*} / c_{\mathrm{b}}\right)$ and $\alpha_{2}=\left(c^{*}-c_{\mathrm{s}}\right) / c_{\mathrm{b}}$ are effective stress coefficients [12], and $\alpha_{i j}^{1}$ and $\alpha_{i j}^{2}$ in Eq. (33) are reduced to Eq. (11).

Introducing Eq. (11) into Eq. (33) gives

$$
\begin{aligned}
& \left(\frac{k_{i j}^{1}}{\mu} p_{1, j}\right)_{, i}=b_{11} \frac{\partial p_{1}}{\partial t}+b_{12} \frac{\partial p_{2}}{\partial t}-\alpha_{1} \frac{\partial e}{\partial t}-\Gamma, \\
& \left(\frac{k_{i j}^{2}}{\mu} p_{2, j}\right)_{, i}=b_{21} \frac{\partial p_{1}}{\partial t}+b_{22} \frac{\partial p_{2}}{\partial t}-\alpha_{2} \frac{\partial e}{\partial t}+\Gamma .
\end{aligned}
$$

Eq. (43) is the fluid flow model for isotropic double porous media with linear elastic deformation, the same as that presented in [12].

\section{Conclusion}

The main purpose of this study is to extend Biot's isothermal, linear poroelastic, coupled fluid-rock, isotropic dual-porosity model to an anisotropic dual-porosity system describing naturally fractured reservoirs. Interpretation of the pore volumetric changes and the associated effective stress law for anisotropic double-porous media are the most critical consideration in the coupling of fluid flow and rock deformation. Compared to the other published models, the proposed model is conceptually consistent between the isotropic and anisotropic dual-porosity concepts in both fluid-flow and geo-mechanical considerations.

\section{Acknowledgment}

This work was supported by the National Natural Science Fund of China.

\section{References}

[1] Barrenblatt GI, Zheltov IP, Kochina IN. Basic concepts in the theory of seepage of homogeneous liquids in fissured rocks. J Appl Math Mech 1960;24:1286-303.

[2] Warren JE, Root PJ. The behavior of naturally fractured reservoirs. Soc Petrol Eng J 1963;Sept:245-55.

[3] Biot MA. General theory of three-dimensional consolidation. J Appl Phys 1941;12:155-64.

[4] Wilson RK, Aifantis EC. On the theory of consolidation with double porosity. Int J Eng Sci 1982;20:1009-35.

[5] Beskos DE, Aifantis EC. On the theory of consolidation with double porosity-II. Int J Eng Sci 1986;24:1697-716.

[6] Bai M, Elsworth D, Roegiers JC. Multiporosity/multipermeability approach to the simulation of naturally-fractured reservoirs. Water Resource Res 1993;29:1621-33. 
[7] Berryman JG, Wang HF. The elastic coefficients of double-porosity models for fluid transport in jointed rock. J Geophys Res 1995;100: 24611-27.

[8] Duguid JO, Lee PCY. Flow in fractured porous media. Water Resource Res 1977;13:558-66.

[9] Valliappan S, Khalili-Naghadeh N. Flow through fissured porous media with deformable matrix. Int J Num Meth Eng 1991;29:1079-94.

[10] Khalili-Naghadeh N, Valliappan S. Flow through fissured porous media with deformable matrix: implicit formulation. Water Resource Res 1991;27:1703-9.

[11] Chen H-Y, Teufel LW. Coupling fluid-flow and geomechanics in dual-porosity modeling of naturally fractured reservoirs. SPE 38884 , presented at the 1997 SPE annual technical conference exhibition, San Antonio. 1997.

[12] Li SQ, Xu BY. Theory model of fluid flow through deformable dual porosity media. J Hydrodynam 2001;16:460-6 (in Chinese).

[13] Zhao Y, Chen M, Zhang GQ. Effective stress law for anisotropic double porous media. Chinese Sci Bull 2004;49: 2327-31.

[14] Carroll MM. An effective stress law for anisotropic elastic deformation. J Geophys Res 1979;84:7510-2.

[15] Zimmerman RW, Chen G, Hadgu T, Bodvarsson GS. A numerical dual-porosity model with semianalytical treatment of fracture/matrix flow. Water Resource Res 1993;29:2127-37. 